\title{
Clinical Effect analysis on indomethacin suppositories prevention of POST-ERCP acute pancreatitis
}

\author{
Liang yanqm, Surgics department, Jjorkers
}

Hospital of He ' Nan Zhongnan Industry Co., Ltcl.nanyang 4 3264, Chma

\begin{abstract}
ObjectiveToinvestigate the clinical effect of indomethacin suppositories prevention of POST-ERCP acute. Pancreatitis20>Methods 180 cases of ERCP were selected from March to September 2015 in ourHospital, all the patients according to the random number table were divided into two groups, there were-cases in the OBsenTation Group and the control group.Hie control group received conventional treatment, the observation group treated with indomethacin on the preventionof the conventional treatment.The serum amylase levels at different time points before and after surgery, acute pancreatitis and hyperamylasemia $</$ b13 $>$

author Units:4732b4Nanyang,Henan Zhongnan Industrial Co., Ltd. Staff Hospital surgery

oftwo groups were compared.ResultsTheserum amylase levels of the observation group and the control group after $2 \mathrm{H}$, after $\mathrm{H}, \mathrm{H}$ PostoperativE were higlier than the preoperative, the degree of elevation of the Obsen ${ }^{\mathrm{T}}$ ation Group was smaller than the conTrol Group, the difference was statistically significance (P 0.05). The incidence of acute pancreatitisand hyperamylasemia of the Obsen 7 ation Group were lower than the control groUp, the difference was statistically significant $(\wedge 0.05)$.conclusionindomethacin can be effective in preventing POST-ERCP acutePancreatitis and reduce the incidence of hyperamylasemia, can significantly lower blood.
\end{abstract}

Keywords: ERCP, pancreatitis, indomethacin suppositories.Prevention

is currently,Endoscopic retrograde cholangiopancreatography(ehcp)because there is no surgery,CreateMinor injury,Surgery short,Low complication rate,Shorten hospitalization time, and so on significantly superiorPoint has been widely used in the diagnosis and treatment of pancreatic duct disease,but due to the patient itselfThe effects of the conditions and the operation techniques of the operator,ERCPmay be issued after surgeryBirth Some complications,pancreatitis toERCPA common complication after surgery.pancreasInflammatory risk greater,easy to cause pancreatic necrosis and systemic multiple organ failure,Severe canendangering the life of the patient ${ }^{[3]}$.to effectively reduceERCPPost-operative acute pancreatitisoccurrence,author on our hospital lineERCPSurgicaltreatment of patients with indomethacin XinXufor preventive treatment,effect better,is reported as follows.

\section{Data and methods}

\subsection{General Information}

This study altogetherISOWpatient,all\%year3\$ |2015Year9Month inOur home lineERCPtreatment between patients, where the maleTheExample,femaleExample,Age

174Thecontinuingmedicaleducation,vol.7,No.

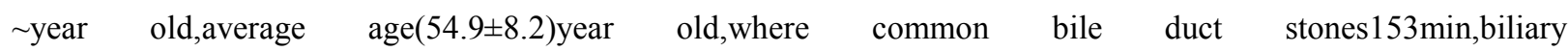
carcinomaexample,Pancreatic Cancer8Gate.randomly divides patients into two groups,Observation Group and controlGroup

\section{Copyright (C)}

This is an open-access article distributed under the terms of the Creative Commons Attribution Unported License

(http://creativecommons.org/licenses/by-nc/4.0/), which permits unrestricted use, distribution, and reproduction in any medium, provided the original work is properly cited.

eachgate,age of two groups of patients-,Gender general data differences by statistical analysisNo statistically 
significant $(\mathrm{P}>0.05)$.iscomparable.All pregnancies are excluded from the study and thelactatingwomen.exclude patients with heart or liver or kidneyinsufficiency,Exclude the researchpatients with drug allergy.All patients have informed consent to the study andsigninformed consent.

\subsection{Method}

Both groups of patients were given a fast,rehydration,support, such as general treatment,Observation Groupusing indomethacin for preventive treatment based on conventional therapy,before $0.5 \mathrm{~h}$,after Operationlhvia rectum indomethacin suppositorym $\mathrm{N}_{\mathrm{LG}}$.control group only give regular treatment,not givingprophylactic medication.

\subsection{Diagnostic Criteria?}

observe clinical manifestations of patients,detects its blood amylase level,Determines whether the patient isoccurrence of acute pancreatitis or high amylase syndromespecific criteria are as follows:ERCPCheckor postoperative serum amylase is significantly higher and higher than normal high-limit.over3Times,longer thanH,with abdominal pain, Tenderness, Vomit,SendHot Clinical Performance,Symptoms of abdominal pain symptoms can be diagnosed as aggravatedERCPoperationpost-pancreatitis;after3HSerum amylase is significantly higher than normal high-limitand rising more than2Times,longer thanH,can be diagnosed as high post operation"bloody enzyme".

\section{4 statistical analysis}

withSPSS17.0Statistical software statistic analysis of all data,which is countedQuantity data is represented as(average \pm standard deviation)formGroup at different timesPoint comparison withFvalidation,22 Comparison of time points in group and comparison between two groups useFValidation,Comparison of Count data with $\mathrm{x}^{2}$ Validation, Test level $=0.05$.

\section{Results}

\subsection{Comparison of serum amylase levels between the two groups at different points in time}

observation of the serum amylase level compared with the control group was not statistically significant in Chua group(corpse $>0.05$ ); Post-operation2h-, and1I_,1Ithe level of serum amylase in is compared to the preoperative ratioappears significantlyhigher,and the control group is more elevated,Two sets of differences statisticsLearn meaning(corpse $<0.05)$.

\subsection{Comparison of the occurrence of acute pancreatitis and high amylase in two groups ofpatients}

The incidenceof acute pancreatitis and high levels of amylase in the observation group were lower than that of the controlGroup, difference between the two groups is statistically significant $(\mathrm{P}<0.05)$.See table2.

\section{Discussion}

ERCPis an important basis for imaging diagnosis of biliary and pancreatic diseases and important treatment of biliary and pancreaticdiseasesmeasures,butehcpbelongs to traumatic technology,pancreatitis isERCPmost common after surgeryA complication of,with postoperativeH severePain,and amylase above normal

value High-limit3times the primary clinical manifestation.ERCPoccurrence of postoperative acute pancreatitis not onlyMake the patient's pain significantly increased,delay treatment,Extended hospitalization time,and alsoIncreased cost of treatment,increasedfinancial burden for patients $^{\mathrm{LI}]}$,and its life securitymaybe compromised,This partly makesERCPThis technique in clinical should bewith restricted,therefore,takes a valid approach to theERCPPre-operative complications forAnti-disease treatment and prognosis of patients have important clinical significance $^{\mathrm{lb}]}$.

indomethacin isCOX-1andCOX-2a non-selective inhibitor,can be suppressed bymaking phospholipaseA2 
(PLA2),to adjust the media before inflammation,Tomake the acute pancreasInflammationEarly Inflammatory response mitigation ${ }^{[7]}$.theRectal application of indomethacin suppository can quickly start, $\operatorname{In}[\sim] m m u p t o$ Peak,and fully bio-exploited,drug in rectum, most of theblood goes directly through the liver,accounts for upto $50 \% \sim 75 \%$. Theuse of suppositories in the study can significantly reduce the damage to the gastric mucosa by the drug.,avoid adverse reactions such as drug-induced gastrointestinal bleeding,This method is cost-effective,Simple Operationmultiline ${ }^{\wedge}$.This result shows that,,the incidenceof acute pancreatitis and high amylase bloodincidence is lower than control group,difference is statistically significant $(\mathrm{P}<0.05)$,Prompt for theindoleindomethacin Effective PreventionERCPPost-operative acute pancreatitis,lowering high amylase bloodincidence.Two groups of different serum amylase levels were significantly higher than before surgeryHigh,and the control group is elevated moresignificantly,difference is statistically significant $($ Corpse $<0.05)$.tips for preventing the use of indomethacin to make thelevel ofserum amylase significantly lower after the operationERCP.

Summary,acute pancreatitis and high amylase areERCPA more commoncomplication,ehcpPostoperative prophylactic indomethacin can cause acute pancreatitis after operation andThe incidence of high amylase is significantly reduced,to increase the level of serum amylase after surgerydecrease,ERCPThe clinical efficacy of is significantly higher.

\section{References}

1. LiuDahuan,Luo Yumacros,LiZhendong, , and so on.NSAIDspreventionERCPPostoperative acute pancreasResearch progress in the Hepatobiliary and Pancreatic surgery journal,2014,2b(5):439-440.

2. ShiHuiping,Poster,WangDecheng,, and so on.ERCPRisk factors and nursing of postoperative pancreatitisinterventionU].Journal of Practical Clinical Medicine,2015,(2):111-113.

3. toperative pancreatitis in thetheFair0]International Journal of Digestive Disorders,2015(3):20o-209.

4. LiuShaofeng, Yuan 鶴,Chento,, and so on.drug preventionERCPPostoperativeAcute pancreasClinical control Study of inflammation-.Journal of Southern Anhui Medical College,2015(3);207-211.

5. Verahong,ChenHaidong,NingLin,, and so on Salvia miltiorrhiza PreventionERCPhigh amylase blood after $28 \mathrm{~b}$. surgeryThe clinical observation of the disease and pancreatitisU].Micro-Chiu Medicine,2014,9(3):2s4-

6. ChengYong..octreotide PreventionERCPClinical efficacy of postoperative pancreatitis[J].China Realwith medicine, $2014(>: 179$.

7. Chen Xiao,TaoLiping,JinQingqing, , and so on.Pre-administration of indomethacin suppository in different time periodsantiERCP therole of postoperative pancreatitis Chinese modern applied pharmacy,2013,((Ten)):11351139.

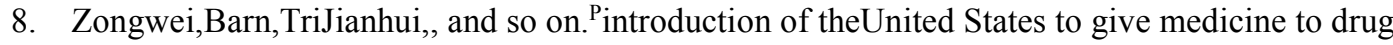
preventionERCPWoodClinical Analysis of post-pancreatitis[J].Shaanxi Medical Journal,2014(S):9s4-9s5.

9. TiTao, Sun Honghai,WangTrijiang, etc..Rectal application of indomethacin in preventionERCP

A study of the role of in postoperative pancreatitis-.Journal of Shandong Medical College,2014\{b):415-417. 